\title{
An accretion disk model for periodic timing variations of pulsars
}

\author{
G. J. Qiao, Y. Q. Xue, R. X. Xu, H. G. Wang, and B. W. Xiao \\ Department of Astronomy, School of Physics, Peking University, Beijing 100871, PR China \\ e-mail: bxyq@vega . bac . pku.edu.cn
}

Received 12 March 2003 / Accepted 23 June 2003

\begin{abstract}
The long-term, highly periodic and correlated variations in both the pulse shape and the rate of slow-down of two isolated pulsars (PSRs) PSR B1828-11 and PSR B1642-03 were discovered recently. This phenomenon may provide evidence for "free precession" as suggested in the literature. Some authors presented various kinds of models to explain this phenomenon within the framework of free precession. Here we present an accretion disk model for this precession phenomenon instead. Under reasonable parameters, the observed phenomenon can be explained by an isolated pulsar with a fossil disk. This may link radio pulsars and anomalous X-ray pulsars (AXPs) and present an indirect evidence for the existence of the fossil disk in nature.
\end{abstract}

Key words. stars: neutron - accretion, accretion disks

\section{Introduction}

Two isolated pulsars (PSR B1828-11 \& B1642-03) present the phenomenon of long-term, highly periodic and correlated variations in both the pulse shape and the slow-down rate (for PSR B1828-11, its variations are best described as harmonically related sinusoids, with periods of approximately 1000 , 500 and 250 days, see Stairs et al. 2000; for PSR B1642-03, its timing residuals exhibit cyclical changes with amplitude varying from 15 to $80 \mathrm{~ms}$ and spacing of maxima varying from 3 to $7 \mathrm{yr}$, see Cordes 1993 and Shabanova et al. 2001). This phenomenon provides the most compelling evidence for precession (Link \& Epstein 2001). Some authors (Jones \& Andersson 2001; Link \& Epstein 2001; Rezania 2002) proposed different models to explain this phenomenon within the framework of free precession. However, further investigations are expected on whether the force of vortices pinning to the stellar crust is strong enough to damp out the free precession of neutron stars (see the conclusion section for details).

The pulsating X-ray binary Her X-1 also shows an unusual kind of periodic timing behavior in the form of an on-off cycle about 35 days. Sarazin et al. (1980) showed that a moderately massive disk $\left(10^{-5}-10^{-2} M_{\odot}\right)$ can cause the precession of the central compact star. Trumper et al. (1983) used the free precession of the neutron star to explain the 35-day period. Qiao \& Cheng (1989) suggested that instead of the free precession of the neutron star, the periodic variation is due to the precession of the accretion disk driven by a normal star.

Send offprint requests to: G. J. Qiao, e-mail: gjn@pku. edu.cn
Actually disk-assisted spin-down of young pulsars has been noted (Menou et al. 2001).

In this paper, we present an accretion disk model to account for the precession of pulsars. The total torque exerted on the pulsar by the fossil disk, which causes the pulsar to precess, is nonzero, due to the asymmetry in pulsar shape and nonalignment between the disk rotation axis and the axis of pulsar moment of inertia. Hence, we present this forced precession model (disk-induced precession), other than the free precession model.

In fact, such a system, i.e. a pulsar with an accretion disk, has been invoked to describe the nature of two kinds of isolated $\mathrm{X}$-ray pulsars: the anomalous X-ray pulsars (AXPs, which have persistent $X$-ray pulsations but few burst) and the soft gamma-ray repeaters (SGRs, which have multiple bursts of soft gamma-rays) (see Xue et al. 2003 for a review). The most remarkable difference between these two kinds of radio-quiet $\mathrm{X}$-ray pulsars and the radio pulsars is that their X-ray luminosities (usually $L_{\mathrm{X}} \sim 10^{35} \mathrm{ergs} / \mathrm{s}$ ) are several orders of magnitude higher than their rotational energy loss rates. To explain this and other exotic facts, two alternative models, viz. the magnetar models (in which the pulsar is suggested to be a neutron star with super-strong magnetic field) and the accretion disk models, were proposed (see Xue et al. 2003 for a review). Under the framework of the accretion disk model, a problem would come if the precession of radio pulsars (e.g. PSR B1811-11 and B1642-03) was really caused by the accretion disk: why do some pulsars with disks behave as ordinary radio pulsars while others behave as AXPs/SGRs? In this paper, we discuss this problem in Sect. 3 after presenting the detailed calculation in Sect. 2. 


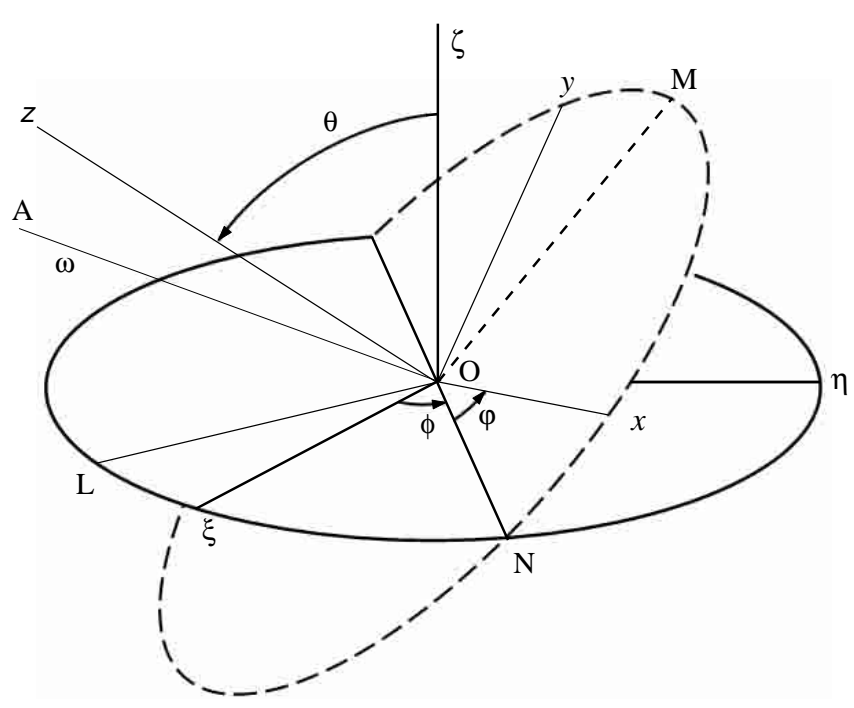

Fig. 1. The geometry and parameters for the calculations. In our calculation, the rotation axis $O A$ is aligned with the principal axis $O z$ of moment of inertia of the neutron star.

\section{Pulsar precession caused by an accretion disk}

\subsection{Geometry and parameters}

Consider the neutron star as a rotation ellipsoid with the principal moment of inertia $I_{1}=I_{2} \neq I_{3}$ and the corresponding radii $a=a \neq b$. The geometry of the system is shown in Fig. 1: $O-x y z$ is the coordinate system fixed with the neutron star, $O x, O y$ and $O z$ are the principal axes of moment of inertia of the neutron star, the axis $O A$ is the rotation axis of the neutron star (for simplicity, we assume the axis $O z$ is aligned with the axis $O A$ in this paper; in this case, free precession doesn't exist and we can see how the disk makes the neutron star precess more clearly), $O-\xi \eta \zeta$ is the coordinate system fixed with the disk and the disk plane is $O \xi \eta, \theta, \varphi$ and $\phi$ are Euler angles, $\omega$ is the angular velocity of neutron star rotation. We will derive the formulae of the gravitational potential in the neutron star-disk system and the angular velocity of neutron star precession. And then, as an example, we model the precession period of PSR B1818-11. The parameters about PSR B1828-11 are as follows: the rotational period of this pulsar $P=0.405 \mathrm{~s}(\omega=2 \pi / P)$, the period derivative $\dot{P}=6.0 \times 10^{-14} \mathrm{ss}^{-1}$, the precession period $P_{\text {pre }} \approx 1000$ days, the magnetic field $B=5.0 \times 10^{12} \mathrm{G}$ (Stairs et al. 2000).

\subsection{Equations and numerical results}

The gravitational potential between the neutron star and the disk is

$$
\begin{aligned}
V= & -\frac{2 G M M_{0}}{c+d} \\
& \times\left[1-\frac{b^{2}+2 a^{2}}{10 c d}+\frac{3 b^{2}}{20 c d} \sin ^{2} \theta+\frac{3 a^{2}}{20 c d}\left(1+\cos ^{2} \theta\right)\right],
\end{aligned}
$$

where $M, a$ and $b$ are the mass, the equatorial and longitudinal radii of the neutron star, respectively, $M_{0}$ is the mass of the disk, $c$ and $d$ are the inner and outer radii of the disk, respectively.

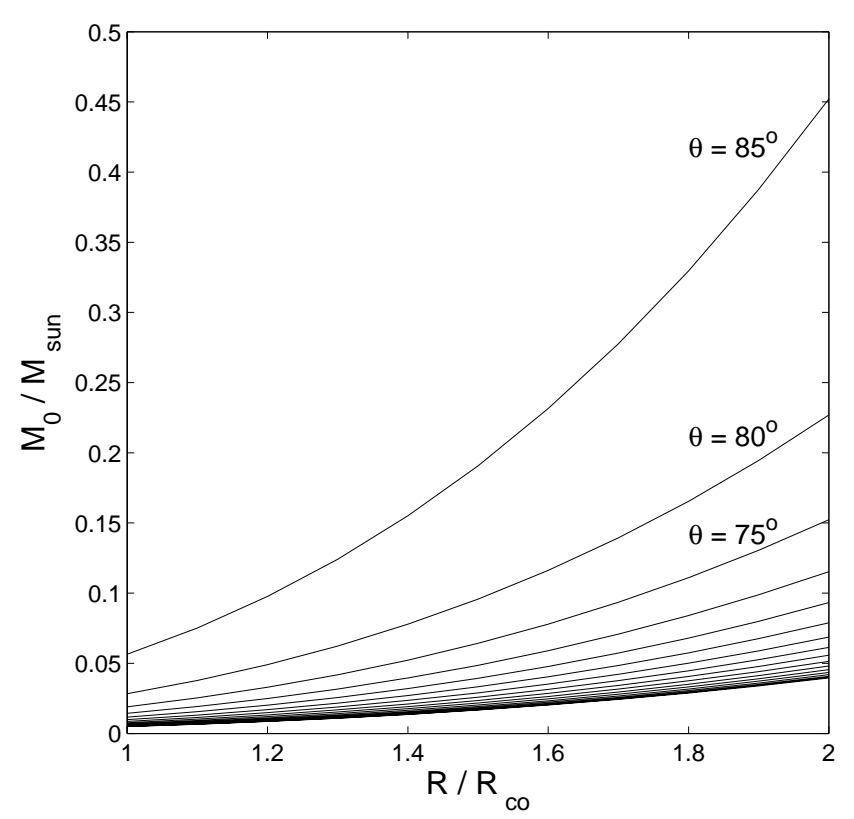

Fig. 2. The relations between $M_{0}$ and $R$ derived from the 1000-day precession period for a group of $\theta$.

The moment of inertia of the neutron star is $I_{\mathrm{z}}=$ $(8 / 15) \pi \rho a^{4} b$, where $\rho=3 M /\left(4 \pi a^{2} b\right)$ is the density of the neutron star. Since the rotation angular velocity of the neutron star $\omega$ is much greater than the precession angular velocity of the neutron star $\dot{\phi}$, the angular momentum of the neutron star can be written as $L_{0} \simeq I_{\mathrm{z}} \omega$. According to the Lagrange's equation $L_{0} \dot{\phi} \sin \theta=-\partial V / \partial \theta$ (Qiao \& Cheng 1989), we get the precession angular velocity of the neutron star:

$\dot{\phi}=-\frac{3 G M_{0} \cos \theta}{2 c d(d+c) \omega}\left(1-\frac{b^{2}}{a^{2}}\right)$.

In terms of the study on the stability of the Maclaurin spheroids, we can obtain the oblateness of this pulsar:

$\epsilon=\frac{a-b}{a}=2.27 \times 10^{-6}$.

For simplification, we assume $c \approx d \approx R$, so that

$\dot{\phi}=-\frac{3 G M_{0} \cos \theta}{4 R^{3} \omega}\left[1-(1-\epsilon)^{2}\right]$.

Figure 2 shows the relations between $M_{0}$ and $R$ derived from the 1000-day precession period for a group of $\theta$. In principle, $M_{0}$ may vary from less than $0.001 M_{\odot}$ to even $0.1 M_{\odot}$ (The amount of the fall-back material $\left(M_{\mathrm{fb}}\right)$ which forms the disk after supernova explosion is uncertain, however, $M_{\mathrm{fb}} \leq 0.1 M_{\odot}$ is supposed to be reasonable, see Chatterjee et al. 2000), and $R$ from about $1 R_{\text {co }}$ (or smaller) to $2 \sim 3 R_{\text {co }}$ when $\theta$ varies within the range of $0 \sim 90^{\circ}$, where $R_{\mathrm{co}}$ is the corotation radius of the disk with the pulsar. Thus, the precession period of the PSR B1828-11 is well elucidated.

\section{Possible evolutionary scenario in the pulsar-disk system}

One may ask where the accretion disk comes from. Usually, a disk around the pulsar can be formed: 1 . by accreting the matter from a companion star in the binary system (approach I); 
2. by the fall-back of the material after a supernova explosion (approach II). In the former process, there are two ways of mass transfer: matter flowing through the first Lagrange point due to the critical Roche Lobe abounding with the companion matter, or by the stellar wind (Nagase 1989).

An interesting issue is that what the pulsar in the pulsar-disk system will behave as, radio pulsar with precession or AXP/SGR? In the accretion model of X-ray binary, accreting process may experience some "phases", e.g. "radio pulsar phase", "sleeping phase", "propeller phase" and "accretion phase" (Illarionov \& Sunyaev 1975; Henriches 1983). The case is suggested to be similar for a pulsar-disk system formed through either approach I or II. The PSR B1828-11, B1642-03, AXPs and SGRs are all isolated "neutron stars". What are the links between them? Here we present a possible series of evolutionary tracks for the pulsar-disk system (cf. Chatterjee et al. 2000 and Henriches 1983).

(a) After the supernova explosion, the newborn neutron star rotates so fast that its magnetospheric radius $R_{\mathrm{m}}$ is much larger than its light cylinder radius $R_{\mathrm{LC}}$. The coupling between the disk and the neutron star is unlikely. The young pulsar initially emits a very strong relativistic wind together with highly energetic magnetic dipole radiation. The pulsar wind and photons blow a cavity in the fall-back flow and no matter can accrete, as is similar in the binary system (Henriches 1983). Thus the neutron star may live in the "radio pulsar phase", i.e. the radio emission can propagate away.

(b) The neutron star slows down as its rotational energy reduces. Its radiation and the high energy particle flow become weakened, thus the cavity decreases and the radio emission is finally quenched. When the "radio pulsar phase" ends, accretion down to the surface of the neutron star is not expected immediately. The so-called "sleeping phase" takes place. This phase lasts until the pulsar rotation slows down enough to make accretion possible.

(c) Subsequently, the neutron star enters the "propeller phase" since accretion onto the neutron star is inefficient due to centrifugal forces acting on the matter. As the rotation slows down further, accretion will be permitted and the neutron star will behave as an accretion-driven $\mathrm{X}$-ray source. Gradually, the system enters a quasi-equilibrium "tracking phase" (happening at the time of $t_{\text {trans }}$ ), in which the spin of the star roughly matches the rotation of the disk at $R_{\mathrm{m}}$. In this phase, the neutron star will be $\mathrm{X}$-ray bright due to accretion. It is possible that the advection-dominated accretion flow (ADAF) will occur at the time of $t_{\mathrm{ADAF}}$, when much of the mass will be ejected prior to reaching the neutron star surface. The X-ray luminosity of the neutron star will decline rapidly with time due to $\dot{M}_{\mathrm{X}}$ (the rate of accretion onto the star surface) diminishing. Therefore, the bright AXPs may exist only between $t_{\text {trans }}$ and $2 t_{\mathrm{ADAF}}$ (about 5000-40000 years).

(d) Finally, accretion ceases since the matter of the disk is exhausted. Thus no accretion-driven X-ray pulsar can be observed and neither can radio emission be emitted owing to the long rotational period of the neutron star (Qiao \& Zhang 1996; Zhang et al. 2000). Consequently, the neutron star may be observed as a dim isolated neutron star, a weak soft X-ray source powered by rotational energy loss or thermal radiation.

\section{Conclusions and discussions}

The accretion disk model for periodic timing variations of pulsars (e.g. PSR B1828-11) can elucidate the observed precession period. According to the analysis of the possible evolutionary scenario discussed above, the PSR B1828-11 may be just in the "radio pulsar phase" before the "sleeping phase" and the "propeller phase". If our model proves to be true, the link between radio pulsars and AXPs may be established and the fossil disk model for AXPs may be strengthened. It also suggests that the neutron star-fossil disk system might exist. Some discussions are listed below.

1. Sarazin et al. (1980) pointed out when the angular momentum of the disk is larger than that of the neutron star, the neutron star will precess due to the effect of the accretion disk and the disk will maintain its orientation. This is just our case for the precession of the PSR B1828-11.

2 . Why is the radio emission expected in our model but not expected in the case of close X-ray binary system? Illarionov $\&$ Sunyaev (1975) suggested that the large optical depth of the stellar wind for free-free absorption hinders the detection of radio pulsars. In our model, a fossil disk is formed and there is no enough stellar wind to absorb the radio emission. A case in point is the PSR B1259-63, which has a $B_{\mathrm{e}}$ companion star with high ellipticity and long orbital period. This neutron star is observed as a radio pulsar when the companion encircles far away from it. But no radio emission is detected when the companion approaches very near the pulsar (Johnston et al. 1992).

3 . The condition when the pulsar radio emission quenches is not clear yet. A possible case may be that the cavity radius becomes smaller than the light cylinder radius (Henriches 1983). In our model, although the corotation radius of the PSR B 1828-11 $R_{\mathrm{co}}=\left(G M / \omega^{2}\right)^{1 / 3} \approx 9.19 \times 10^{7} \mathrm{~cm}$ is one order of magnitude smaller than its light cylinder radius $R_{\mathrm{LC}}=c / \omega=1.93 \times 10^{9} \mathrm{~cm}$, and the disk radius $R$ approaches $2-3 R_{\mathrm{co}}$, this neutron star is still in the "radio pulsar phase". This presents an example for further investigations.

4. We have ignored the angular momentum of the pinned superfluid in the dynamic equations of the calculation. Can the pinning force be strong enough to damp any precession of neutron stars? In principle, detailed computations may determine whether the vortices are pinned, but a definitive conclusion on the nature has not been reached yet due to various uncertainties in the microscopic physics. Based on the calculation of Shaham (1977) and Sedrakian et al. (1999), Stairs et al. (2000) conclude that the torque-free precession of PSR B1828-11 should be damped out due to the dissipation caused by the vortices pinning to the stellar crust on the timescale of several hundred precession periods. Nevertheless, a further consideration of Shaham (1986) indicates an unpinned superfluid in the case of precession. It is also noted by Link \& Cutler (2002), that hydrodynamic forces present in a precessing star are probably sufficient to unpin all of the vortices of the inner crust. In addition, the 35-d periodicity of the accreting binary system (Her X-1) has been interpreted as precession (Shakura et al. 1998, and the references therein), which could be another example of forced precessions. We have therefore a tendency to suggest that, the observation of PSR B1828-11 precession may 
imply that the vertex pinning in this pulsar is much weaker than that predicted previously, at least it is weaker than the possible disk-driven precession effect.

5. In fact, the phenomenon of the secular periodic timing variations, which also exists in many other radio pulsars (e.g. Crab and Vela pulsar), has been investigated both observationally and theoretically (Sedrakian et al. 1999 and the references therein). If a neutron star were a rigid or semirigid solid star, it would precess (Pines \& Shaham 1974); as is mentioned above, the interaction between the superfluid vortices and the stellar crust needs further considerations. Ruderman (2001) pointed out that this is a crucial problem which will raise doubts about canonical descriptions of neutron star structure until it is solved. No matter the precession is torque-free or forced, the neutron star structure still needs to be investigated more. Instead of neutron star, Xu (2003) proposed a solid strange star model, which may be possible and also needs to be investigated more.

6. Our model cannot exclude the possibility of a companion star (instead of a fossil disk) which can produce the precession phenomenon as well. In this case, two limits should be considered: the Roche Limit $d_{\mathrm{R}}$ (if the companion approached the neutron star closer than this limit, it would be torn asunder by tidal forces) and the Instability Limit $d_{\mathrm{I}}$ (if the company strayed farther than this limit, it would escape due to the differential perturbations from other celestial bodies). The details of this case will be studied in another paper.

Acknowledgements. We are grateful to K. J. Lee et al. for their helpful discussions. This work is supported by National Nature Science Foundation of China (10273001, 10073001), and by the Special Funds for Major State Basic Research Projects of China (G2000077602).

\section{References}

Chatterjee, P., Hernquist, L., \& Narayan, R. 2000, ApJ, 534, 373

Cordes, J. M. 1993, in Planets around Pulsars, ASP Conf. Ser., 36, 43 Henriches, H. F. 1983, in Accretion-driven stellar X-ray sources, ed. W. H. G. lewin, \& E. P. J. van den Heuvel (Cambridge Univ. Press), 393

Illarionov, A. F., \& Sunyaev, R. A. 1975, A\&A, 39, 185

Johnston, S., Manchester, R. N., Lyne, A. G., et al. 1992, ApJ, 387, L37

Jones, D. I., \& Andersson, N. 2001, MNRAS, 324, 811

Link, B., \& Cutler, C. 2002, MNRAS, 336, 211

Link, B., \& Epstein, R. I. 2001, ApJ, 556, 392

Menou, K., Perna, R., \& Hernquist, L. 2001, ApJ, 554, L63

Nagase, F. 1989, Publ. Astron. Soc. Japan, 1

Pines, D., \& Shaham, J. 1974, ComAp, 6, 37

Qiao, G. J., \& Cheng, J. H. 1989, ApJ, 340, 503

Qiao, G. J., \& Zhang, B. 1996, A\&A, 306, L5

Rezania, V. 2002, preprint [astro-ph/0205180]

Ruderman, M. 2001 [astro-ph/0109353]

Sarazin, C. L., Begelman, M. C., \& Hatchett, S. P. 1980, ApJ, 238, 129

Sedrakian, A., Wasserman, I., \& Cordes, J. M. 1999, ApJ, 524, 34

Shabanova, T. V., Lyne, A. G., \& Urama, J. O. 2001, ApJ, 552, 321

Shaham, J. 1977, ApJ, 214, 251

Shaham, J. 1986, ApJ, 310, 780

Shakura, N. I., Postnov, K. A., \& Prokhorov, M. E. 1998, A\&A, 331, L37

Stairs, H., Lyne, A. G., \& Shemar, S. L. 2000, Nature, 406, 484

Trumper, J. 1983, MitAG, 58, 7

Xu, R. X. 2003, preprint [astro-ph/0302165]

Xue, Y. Q., Qiao, G. J., Xu, R. X., \& Wang, H. G. 2003, Acta Scientiarum Naturalium, Universitatis Pekinensis, accepted Zhang, B., Harding, A. K., \& Muslimov, A. 2000, ApJ, 531, L135 